\title{
A prospective evaluation of prevalence of microbial flora and significance of intraoperative Peritoneal culture of fungus in perforation Peritonitis
}

\author{
Goyal $\mathbf{V}^{1}$, Pathania $\mathbf{S}^{2}$, Goyal $\mathbf{V}^{3}$, Sandhu HPS ${ }^{4}$ \\ ${ }^{1}$ Dr. Vikas Goyal, Assistant Professor, Department of Surgery, Affliated with G.G.S. Medical College, Faridkot, Punjab, \\ India. ${ }^{2}$ Dr Sumeet Pathania, Junior Resident, previously, Department of Surgery, Affliated with G.G.S. Medical College, \\ Faridkot, Punjab, India. ${ }^{3}$ Dr. Vishal Goyal, Medical Officer, ${ }^{4}$ Dr. H.P.S. Sandhu, Professor, Incharge Surgery 2, Affliated \\ with G.G.S. Medical College, Faridkot, Punjab, India.
}

Address for correspondence: Dr. Vikas Goyal, Email: drvikasgoyal2006@yahoo.com

\begin{abstract}
Objective: To study the prevalence of intraabdominal flora and significance of intraoperative peritoneal fluid culture of fungus in patients with perforation peritonitis. Study design: Prospective study. Methods: In this study, we analyzed 91 patients of gastrointestinal perforation admitted during the period from January 2011 to July 2012 in Department of Surgery. Any patient undergoing exploratory laparotomy for gastrointestinal perforation above 5 years was included. Intraoperative abdominal fluid samples were collected and cultured. Results: Ninety one patients of gastrointestinal perforation were studied. The mean presenting age was $35.21+12.87$ years. Gastro duodenal perforation was the commonest $(48.35 \%), 34.07 \%$ were having ileal perforation, $6.59 \%$ were having appendicular perforation, $5.49 \%$ were having jejunal and 5.49\% had large bowel perforation. Out of 91 patients, $79(86.8 \%)$ patients showed growth of either bacteria or fungus and in $12(13.2 \%)$ patient's culture was sterile. Gram positive cocci was found in 46.1\%, E.Coli in 40.65\%, Klebsiella in 14.28\%, Enterobacter in $1.098 \%$ and Pseudomonas in $1.089 \%$ of patients. Fungal growth was seen in $48.3 \%$ of patients. Patients with fungal positive culture had superficial surgical site infection in $77.27 \%$, deep surgical site infection $59.09 \%$ and residual abscesses formation in $27.27 \%$ of cases. Patient with no growth of fungus have superficial site infection in $40 \%$, deep surgical site infection in $25.71 \%$ and residual abscess formation in $5.71 \%$. Conclusion: Positive peritoneal fungal co-infection is a bad prognostic factor and a significant risk factor for adverse outcome in perforation peritonitis.
\end{abstract}

Keywords: Perforation Peritonitis, Fungus.

\section{Introduction}

Peritonitis is inflammation of peritoneum and peritoneal cavity and is most commonly due to localized or generalized infection. Perforation peritonitis is the most common surgical emergency in India. The management of peritonitis continues to have high morbidity and mortality inspite of improved surgical techniques and antibiotics [1]. Until recently the leading pathogens associated with secondary peritonitis were gram negative and anaerobic bacteria. Fungal infection has become more common in recent years especially in critically ill patients in intensive care [2]. Higher

Manuscript received: $8^{\text {th }}$ Nov 2015

Reviewed: $19^{\text {th }}$ Nov 2015

Author Corrected: $30^{\text {th }}$ Nov 2015

Accepted for Publication: $18^{\text {th }}$ Dec 2015 incidence of fungal isolates has been reported in gastroduodenal perforations [3]. In this study, we took intraoperative cultures of gastrointestinal perforation peritonitis patients and studied the prevalence of microflora of intraoperative peritoneal aspiration.

\section{Aims and Objectives}

The study was undertaken to study the prevalence of intraabdominal flora in patients with gastrointestinal perforation.

To study the significance of intraoperative peritoneal fluid culture of fungus and establish the indications for treatment in patients with perforation peritonitis. 


\section{Material and Methods}

In this study, we analyzed 91 patients of gastrointestinal perforation admitted during the period from January 2011 to July 2012 in Department of Surgery in our medical college situated in backward area. Any patient undergoing exploratory laparotomy for gastrointestinal perforation above 5 years was included. Patients who presented with primary peritonitis or perforation due to trauma were excluded.

Microbiological Sampling: The intraoperative samples of abdominal fluid were collected during laparotomy in sterile container using all sterile precautions and samples were immediately transferred to microbiology laboratory. In case of delay, samples were kept at $4^{0} \mathrm{C}$ till the time of transfer. In microbiology laboratory culture were performed after (1) Direct smear examination (2) Gram staining of peritoneal fluid.

For bacterial culture sample were inoculated on blood agar and MacConkey agar plates and were incubated at $37^{\circ} \mathrm{C}$ for 24 hours. For fungal cultures the specimen were inoculated on Sabouraud dextrose agar and were examined after 48 hours of incubation. Any bacterial growth appearing on blood or MacConkey agar medium was studied for colony characters. Bacterial smear made for the colony was gram stained and bacterial motility was examined. Final identification of bacteria was made after doing biochemical tests. (1) Catalase (2) Indole (3) Methyl red (4) Voges Proskaeur (5) Citrate Utilization (6) Oxidase (7) Urease.

For identification of the fungal growth, fungal smear were examined in lactophenol cotton blue. Fungal growth showing budding yeast cells (candida) was subjected to germ tube test. For doing germ test, single yeast colony was inoculated in $1 \mathrm{ml}$ of human serum and incubated at $37^{\circ} \mathrm{C}$ for 2 hours.

Clinical outcome in fungus positive and fungus negative patients was compared on basis of surgical site infection, residual abscess, I.C.U. stay, hospital stay and mortality.

\section{Observations}

Ninety one patients of gastrointestinal perforation were studied. The mean presenting age was $35.21+12.87$ years. The maximum patients fell in age group of 26-35 years (38.46\%).85.71\% were male and $14.29 \%$ were female. In our study, only eight patients presented within 24 hours of onset of symptoms. Majority of patients (51.65\%) presented within 2-3 days of onset of symptoms. $87.9 \%$ of patients had history of preoperative use of antibiotics. On general physical examination preoperatively, $86.81 \%$ had tachycardia, $39.56 \%$ had fever, and $26.37 \%$ had hypotension, $90.11 \%$ had tenderness and $85.71 \%$ had abdominal distension, $97.81 \%$ had abdominal guarding. $93.41 \%$ patients showed air under diaphragm on X-ray abdomen. Gastro duodenal perforation was the commonest (48.35\%), 34.07\% were having ileal perforation, $6.59 \%$ were having appendicular perforation, $5.49 \%$ were having jejunal and $5.49 \%$ had large bowel perforation.

Out of 91 patients, $79(86.8 \%)$ patients showed growth of either bacteria or fungus and in $12(13.2 \%)$ patient's culture was sterile. Only bacterial growth was obtained in 35(38.46\%), both bacteria and fungus was obtained in $36(39.50 \%)$ patients and $8(8.79 \%)$ patients had growth of fungus only (Table 1$)$.

Table- 1: Microbial flora according to site of perforation

\begin{tabular}{|l|l|l|l|l|l|l|}
\hline Perforation & E.Coli & Klebsiella & $\begin{array}{l}\text { Gram +ve } \\
\text { cocci }\end{array}$ & Fungi & Pseudomonas & Enterobacter \\
\hline $\begin{array}{l}\text { Gastroduodenal } \\
\mathrm{n}=44\end{array}$ & $20.4 \%$ & $6.8 \%$ & $50.4 \%$ & $70.4 \%$ & - & - \\
\hline $\begin{array}{l}\text { Small gut } \\
\mathrm{n}=36\end{array}$ & $61.1 \%$ & $22.2 \%$ & $38.8 \%$ & $33.3 \%$ & - & - \\
\hline $\begin{array}{l}\text { Appendicular } \\
\mathrm{n}=6\end{array}$ & $50 \%$ & $16.6 \%$ & $33.3 \%$ & - & - & $16.6 \%$ \\
\hline $\begin{array}{l}\text { Large gut } \\
\mathrm{n}=5\end{array}$ & $60 \%$ & $20 \%$ & $40 \%$ & $20 \%$ & $20 \%$ & - \\
\hline
\end{tabular}


Among bacterial culture, gram positive cocci was found in $46.1 \%$, E.Coli in $40.65 \%$, Klebsiella in $14.28 \%$, Enterobacter in $1.098 \%$ and Pseudomonas in $1.089 \%$ of patients. Fungal growth was seen in $48.3 \%$ of patients. Out of 91 patients, 8 $(8.79 \%)$ patients died. Out of 80 patients, in whom preoperatively antibiotics were used in $42(52.5 \%)$ patients, growth of fungus was obtained.

Patient with fungal positive culture had superficial surgical site infection in $77.27 \%$, deep surgical site infection $59.09 \%$ and residual abscesses formation in $27.27 \%$ of cases. Patient with no growth of fungus have superficial site infection in $40 \%$, deep surgical site infection in $25.71 \%$ and residual abscess formation in $5.71 \%$ (Table 2).

Table- 2: Post-operative complications

\begin{tabular}{|l|l|l|}
\hline Types of complications & No. of patients & Percentage \\
\hline Superficial surgical site infection & 48 & $52.75 \%$ \\
\hline Deep surgical site infection & 35 & $38.46 \%$ \\
\hline Residual abscess & 15 & $16.48 \%$ \\
\hline LRTI ( crepts) & 11 & $12.09 \%$ \\
\hline Pleural effusion & 13 & $14.29 \%$ \\
\hline Faecal fistula & 7 & $7.69 \%$ \\
\hline
\end{tabular}

So patients with fungal positive culture have high incidence of superficial surgical site infection and residual abscess formation compared to fungus negative culture and the $\mathrm{p}$ value is also statistically significant (Table 3 ).

Table- 3: Relationship of fungal culture to surgical site infection and residual abscess

\begin{tabular}{|l|c|c|c|c|c|}
\hline Variables & \multicolumn{2}{|c|}{ Fungal positive } & \multicolumn{2}{c|}{ Fungal negative } & p-value \\
\hline & \multicolumn{2}{|c|}{ No. \%age } & \multicolumn{2}{c|}{ No. \%age } & \\
\hline S.S. infection & 34 & 77.27 & 14 & 40.00 & 0.00766 \\
\hline D.S. infection & 26 & 59.09 & 9 & 25.71 & 0.00871 \\
\hline Residual infection & 12 & 27.27 & 2 & 5.71 & 0.03931 \\
\hline
\end{tabular}

Patient with fungal positive culture have high incidence of I.C.U stay, hospital stay and mortality compared to fungal negative culture and the $\mathrm{p}$ value is also statistically significant (Table 4).

Table 4: Relationship of Fungal Culture to ICU Stay, Hospital Stay and Mortality

\begin{tabular}{|l|c|c|c|c|c|}
\hline Variables & \multicolumn{2}{|c|}{ Fungal Positive } & \multicolumn{2}{c|}{ Fungal Negative } & p-value \\
\hline ICU STAY & 18 & 40.91 & 5 & 14.29 & 0.00997 \\
\hline H. STAY & 32 & 72.73 & 11 & 31.43 & 0.00705 \\
\hline Mortality & 7 & 15.21 & 1 & 2.86 & 0.08638 \\
\hline
\end{tabular}

\section{Discussion}

Perforation peritonitis in our study is most commonly seen in young patients (35.21years) which corresponds to other indian study [4] but slightly less than previous studies from the west $[5,6]$. Male patients were more commonly affected by six times than female patients. Presenting symptoms were abdominal distension in $85.7 \%$, tachycardia in $86.81 \%$ of patients. The commonest site of perforation in our study was gastroduodenal $48.35 \%$ as compared to west where distal gastrointestinal tract perforations are more common [4,7].
Our series showed that in case of gastroduodenal perforation, out of 44 patients, fungus growth was obtained in maximum number of $70.4 \%$ followed by gram positive cocci $50.4 \%$, E. Coli $20.4 \%$ and Klebisella $6.8 \%$. According to Ruiter and Shan fungus growth was obtained in $41 \%$ and $43.4 \%$ patients [ 3,8$]$. In our study, fungus growth was obtained in slightly more number of patients probably due to more use of preoperative antibiotics $(87.9 \%)$ and late presentation of patients for the surgery. In patients with small gut perforation, E. coli was the most common organism seen in $61.1 \%$, gram positive cocci in $38.8 \%$ patients, 
fungus growth in $33.3 \%$ and Klebsiella in $22.2 \%$ patients. Similar results were seen in Ruiter et al study which showed gram negative bacteria in $46.3 \%$ of patients, gram positive cocci in $43.9 \%$ and fungus in $34.1 \%$ of patients. In patients with appendicular perforation, E. Coli was seen in $50 \%$ patients, gram positive cocci in $33.3 \%$, Klebsiella in $16.6 \%$ and enterobacter in $16.6 \%$. Similar results were found ruiter et al study in which gram negative was found in $77.8 \%$ and gram positive in $33.3 \%$. No fungal growth was seen in appendicular perforations. In patients with large gut perforation, E.Coli growth was seen in $60 \%$ patients, gram positive cocci in $40 \%$ patients, Klebisella in $20 \%$, Pseudomonas in $20 \%$ and fungus in $20 \%$.

In our study, the most common complication was superficial surgical site infection seen in $52.75 \%$, and deep surgical site infection in $38.46 \%$ residual abscess in $16.48 \%$, lower respiratory tract infection in $12.09 \%$, pleural effusion in $14.29 \%$ and faecal fistula in $7.69 \%$. Mortality was $8.79 \%$ in our study. Similar results were seen in Stephen et al, Rajinder jhobta and Shaida Parveen $[4,9,10]$.

Patients with fungal positive culture had superficial surgical site infection in $77.27 \%$, deep surgical site infection in $59.09 \%$ and residual abscess formation in $27.27 \%$ of cases. Patients with no growth of fungus have superficial surgical site infection in $40 \%$, deep surgical site infection in $25.71 \%$ and residual abscess formation in $5.71 \%$. Shan et al shows superficial surgical site infection in $27 \%$ of patients, deep surgical site infection in $38.1 \%$ and residual abscess in $15.9 \%$ of fungus culture positive patients. Patients with fungal positive culture had ICU stay of more than 5 days in $40.91 \%$ of patients, hospital stay of more than 15 days in $72.73 \%$ of patients and mortality of $15.21 \%$. Similar results were reported by studies done by Y-Shan et al, Adavit Parkash and Sandven et al $[1,3,11]$.

\section{Conclusion}

Gastroduodenal perforation is the major cause of perforation peritonitis followed by small gut perforation. E. Coli is the most common pathogen in small gut, appendix, large gut perforation peritonitis cases. Fungus (Candida) is the most common micro organism in gastric perforation. Patients with fungal positive culture had higher incidence of surgical site infection, residual abscess formation, longer ICU stay, longer hospital stay and higher mortality rates in comparison to fungal culture negative patients and results were statistically significant. So we conclude that positive peritoneal fungal co-infection is a bad prognostic factor and a significant risk factor for adverse outcome in perforation peritonitis.

\section{Funding:Nil. Conflict of interest: Nil. Permission for IRB: Yes}

\section{Bibliography}

1. Prakash A, Sharma D, Saxena A, Somashekar U, Khare N, Mishra A, Anvikar A. Effect of Candida infection on outcome in patients with perforation perito nitis. Indian J Gastroenterol. 2008 May-Jun;27(3):1079.

2. Calandra T, Bille J, Schneider R, Mosimann F, Francioli P. Clinical significance of Candida isolated from peritoneum in surgical patients. Lancet. 1989 Dec 16;2(8677):1437-40.

3. Shan YS, Hsu HP, Hsieh YH, Sy ED, Lee JC, Lin $\mathrm{PW}$. Significance of intraoperative peritoneal culture of fungus in perforated peptic ulcer. Br J Surg. 2003 Oct;90(10):1215-9.

4. Jhobta RS, Attri AK, Kaushik R, Sharma R, Jhobta A. Spectrum of perforation peritonitis in India--review of 504 consecutive cases. World J Emerg Surg. 2006 Sep 5;1:26.

5. Hunt JL. Generalized peritonitis. To irrigate or not to irrigate the abdominal cavity. Arch Surg. 1982 Feb;117(2):209-12.

6. Mosdell DM, Morris DM, Voltura A, Pitcher DE, Twiest MW, Milne RL, Miscall BG, Fry DE. Antibiotic treatment for surgical peritonitis. Ann Surg. 1991 Nov;214(5):543-9.

7. Dandapat M.C, Mukherjee. Gastrointestinal perforation. IJS : 1991;53:5;188-193.

8. de Ruiter J, Weel J, Manusama E, Kingma WP, van der Voort PH. The epidemiology of intraabdominal flora in critically

ill patients with secondary and tertiary abdominal sepsis Infection. 2009 Dec;37(6):522-7. doi:

$10.1007 / \mathrm{s} 15010-009-8249-6$.

9. Shan YS, Hsu HP, Hsieh YH, Sy ED, Lee JC, Lin PW. Significance of intraoperative peritoneal culture of 
fungus in perforated peptic ulcer. Br J Surg. 2003 Oct;90(10):1215-9.

10. Shahida Parveen Afridi, Faiza Malik, Shafiq UrRahman , Shahid Shamim and Khursheed A Samo. Spectrum of perforation peritonitis in Pakistan: 300 cases Eastern experience. World Journal of Emergency Surgery 2008 Nov 8; 3:31.doi: 10.1186/1749-7922-331.
11. Stephen M, Loewenthal J. Continuing peritoneal lavage in high-risk peritonitis. Surgery. 1979 Jun;85(6):603-6.

12. Sandven P, Qvist H, Skovlund E, Giercksky KE; NORGAS Group and the Norwegian Yeast Study Group. Significance of Candida recovered from intraoperative specimens in patients with intraabdominal perforations. Crit Care Med. 2002 Mar;30(3):541-7.

\section{How to cite this article?}

Goyal V, Pathania S, Goyal V, Sandhu HPS. A prospective evaluation of prevalence of microbial flora and significance of intraoperative Peritoneal culture of fungus in perforation Peritonitis. Int J Med Res Rev 2015;3(11):1317-1321. doi: 10.17511/ijmrr.2015.i11.239. 